\title{
Monolayers of $\mathrm{MoS}_{2}$ on $\mathrm{Ag}(111)$ as decoupling layers for organic molecules: resolution of electronic and vibronic states of TCNQ
}

\author{
Asieh Yousofnejad, Gaël Reecht ${ }^{\star}$, Nils Krane, Christian Lotze and Katharina J. Franke
}

\section{Full Research Paper}

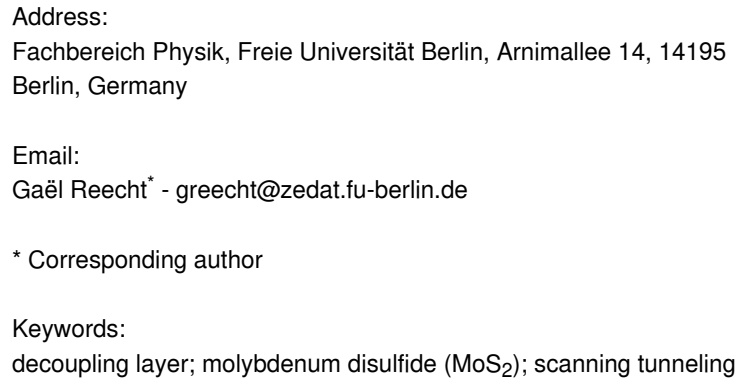

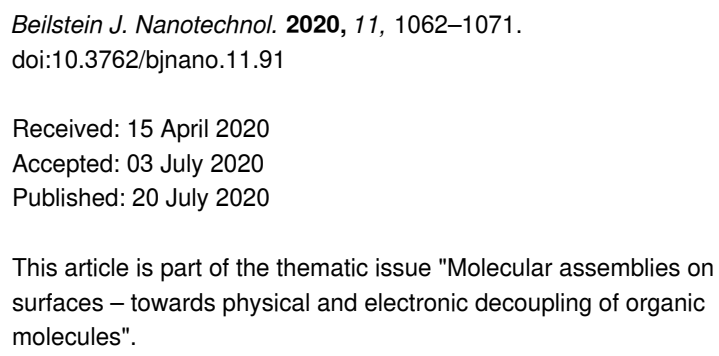

Guest Editor: S. Maier

(C) 2020 Yousofnejad et al.; licensee Beilstein-Institut. License and terms: see end of document.

\begin{abstract}
The electronic structure of molecules on metal surfaces is largely determined by hybridization and screening by the substrate electrons. As a result, the energy levels are significantly broadened and molecular properties, such as vibrations are hidden within the spectral line shapes. Insertion of thin decoupling layers reduces the line widths and may give access to the resolution of electronic and vibronic states of an almost isolated molecule. Here, we use scanning tunneling microscopy and spectroscopy to show that a single layer of $\mathrm{MoS}_{2}$ on $\mathrm{Ag}(111)$ exhibits a semiconducting bandgap, which may prevent molecular states from strong interactions with the metal substrate. We show that the lowest unoccupied molecular orbital (LUMO) of tetracyanoquinodimethane (TCNQ) molecules is significantly narrower than on the bare substrate and that it is accompanied by a characteristic satellite structure. Employing simple calculations within the Franck-Condon model, we reveal their vibronic origin and identify the modes with strong electron-phonon coupling.
\end{abstract}

\section{Introduction}

When molecules are adsorbed on metal surfaces, their electronic states are strongly perturbed by hybridization, charge transfer and screening [1-4]. These effects lead to a broadening and shift of the molecular resonances [5]. Often the molecular functionality is also lost due to these interactions [6]. However, addressing individual molecules in devices or by single-mole- cule spectroscopy as offered in a scanning tunneling microscope, requires a metal electrode. To (partially) preserve the molecular properties the molecule-electrode coupling has to be properly designed. An elegant way is to clamp the molecule between electrodes via single-atom bonds at opposing sites of the molecule while the molecule is freely hanging between the elec- 
trodes [7-10]. While these configurations give access to important transport properties [11-13], they do not allow for imaging molecular properties with intramolecular resolution [14]. The latter requires the molecules to be flat lying on a surface. To decouple such flat-lying molecules from a metal, thin insulating layers have been engineered, ranging from ionic salts [15,16], over oxides [17-19], nitrides [20], and molecular layers $[21,22]$ to $2 \mathrm{D}$ materials, such as graphene $[23,24]$, and hexagonal boron nitride [25].

The most recent development of decoupling layers made use of the in situ fabrication of single layers of transition metal dichalcogenides on metal surfaces. A monolayer of $\mathrm{MoS}_{2}$ on $\mathrm{Au}(111)$ provided very narrow molecular resonances, close to the thermal resolution limit at $4.6 \mathrm{~K}$ [26]. The exquisite decoupling efficiency has been ascribed to a combination of its rather large thickness of three atomic layers, its electronic bandgap, and its non-ionic nature. Together, these properties prohibited fast electronic relaxations into the metal and coupling to phonons, which otherwise led to lifetime broadening [27,28].

The electronic properties of $\mathrm{MoS}_{2}$ on a metal surface are not the same as those of a free-standing monolayer. Both theory and experiment have found considerable hybridization of electronic states at the interface [29]. As a consequence, the bandgap is narrowed. Instead of the predicted bandgap of $2.8 \mathrm{eV}$ of the free-standing layer [30,31], the bandgap of the hybrid structure amounts to only approx. $1.7 \mathrm{eV}$ [29]. Interestingly, the states at the $K$ point are much less affected than the states at the $\Gamma$ point. Hence, the system remains promising for optoelectronic devices with selective access to the spin-orbit-split bands at $K$ and $K^{\prime}$ by circularly polarized light [32].

The potential as decoupling layer for molecules may become even more appealing by the fact that monolayers of transition metal dichalcogenides can be grown in situ on different metal surfaces, where the precise hybridization and band alignment depend on the nature of the substrate [33]. One may thus envision tuning the bandgap alignment for decoupling either the lowest unoccupied (LUMO) or the highest occupied molecular orbital (HOMO) of the molecules.

While $\mathrm{MoS}_{2}$ on $\mathrm{Au}(111)$ has already been established as an outstanding decoupling layer [26], we will now explore this potential for $\mathrm{MoS}_{2}$ on a $\operatorname{Ag}(111)$ surface. In agreement with the band modifications of $\mathrm{WS}_{2}$ on $\mathrm{Au}(111)$ and $\mathrm{Ag}(111)$, we find that the bandgap remains almost the same, albeit shifted to lower energies [33]. As a test molecule we chose tetracyanoquinodimethane (TCNQ). Due to its electron-accepting character, this choice will allow us to detect a negative ion resonance within the bandgap of $\mathrm{MoS}_{2}$. We will show that the LUMO is indeed decoupled from the metallic substrate as we can detect a narrow line width followed by a satellite structure. We can reproduce this fine structure by simulating the vibronic states of the gasphase molecule.

\section{Results and Discussion}

We have grown monolayer islands of $\mathrm{MoS}_{2}$ on an atomically clean Ag(111) surface, which had been exposed to sputtering-annealing cycles under ultrahigh vacuum before. The growth procedure was adapted from that of $\mathrm{MoS}_{2}$ on $\mathrm{Au}(111)[34,35]$, with Mo deposition on the surface in a $\mathrm{H}_{2} \mathrm{~S}$ atmosphere of $5 \cdot 10^{-5}$ mbar, while the sample is annealed to $800 \mathrm{~K}$. TCNQ molecules were deposited on the as-prepared sample held at $230 \mathrm{~K}$. The sample was then cooled down and transferred to the scanning tunneling microscope (STM). All measurements were performed at 4.6 K. Differential conductance $(\mathrm{d} I / \mathrm{d} V)$ maps and spectra were recorded with a lock-in amplifier at modulation frequencies of $812-921 \mathrm{~Hz}$, with the amplitudes given in the figure captions.

\section{Characterization of single-layer $\mathrm{MoS}_{2}$ on $\mathrm{Ag}(111)$}

Figure 1a presents an STM image of the $\mathrm{Ag}(111)$ surface after the growth of $\mathrm{MoS}_{2}$ as described above. We observe islands with tens to hundreds of nanometer diameter and of $2.3 \pm 0.2 \AA$ apparent height (inset of Figure 1). The apparent height is much smaller than the layer distance in bulk $\mathrm{MoS}_{2}$ [36] due to electronic structure effects, but in agreement with a single layer of $\mathrm{MoS}_{2}$ on a metal surface [34]. The islands exhibit a characteristic hexagonal pattern reflecting a moiré structure, which results from the lattice mismatch between the $\mathrm{Ag}(111)$ surface and $\mathrm{MoS}_{2}$ (Figure 1b). Areas with large apparent height correspond to domains in which the $\mathrm{S}$ atoms sit on top of $\mathrm{Ag}$ atoms, whereas the lower areas represent two different hollow sites (fcc or hcp stacking) of the $\mathrm{S}$ atoms on the Ag lattice. The most abundant moiré periodicity amounts to approx. $3.3 \pm 0.1 \mathrm{~nm}$. This value is similar to the one observed for $\mathrm{MoS}_{2}$ on $\mathrm{Au}(111)$ [29,32,34,37].

Given the similar lattice constants of $\mathrm{Au}(4.08 \AA)$ and $\mathrm{Ag}$ (4.09 $\AA$ ), a locking into a similar superstructure at the metal- $\mathrm{MoS}_{2}$ interface is not surprising. However, occasionally, we also observe moiré patterns with lattice constants of $3.6 \pm 0.1$ and $3.0 \pm 0.1 \mathrm{~nm}$, and different angles between $\mathrm{MoS}_{2}$ and the $\operatorname{Ag}(111)$ lattice. This indicates shallow energetic minima of the lattice orientations. Atomically resolved STM images (Figure 1c) reveal the expected S-S distance of $3.15 \AA$ in the top layer [36,39-41].

For an efficient decoupling of a molecule from the substrate, the interlayer must provide an electronic bandgap. As the moiré 

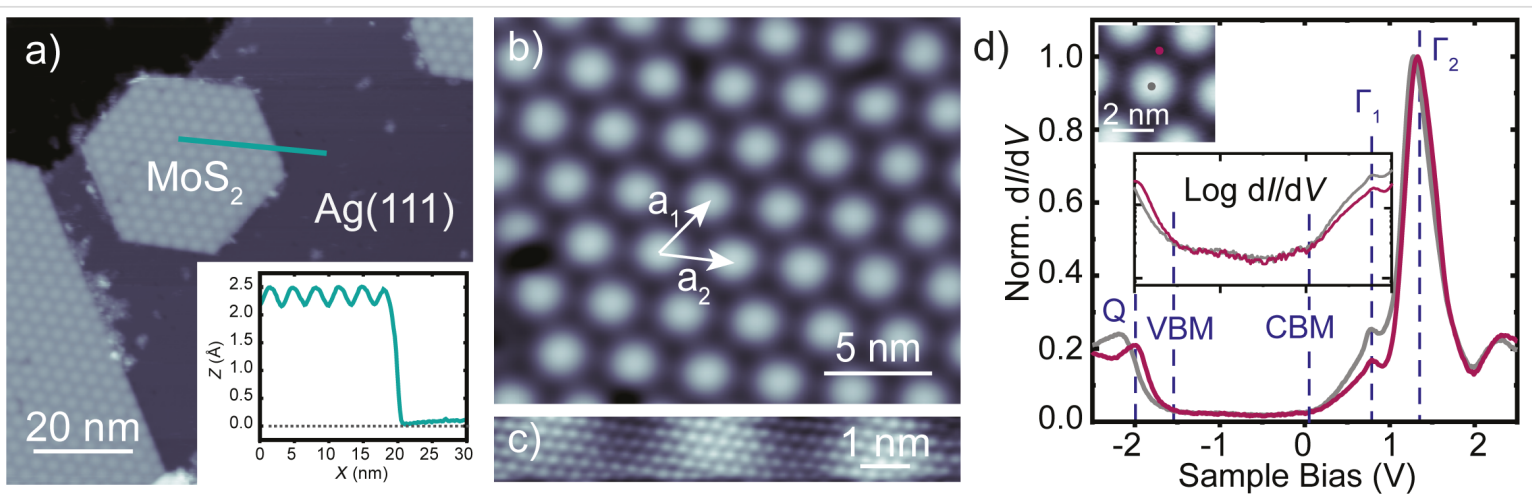

Figure 1: a) STM topography of $\mathrm{MoS}_{2}$ on $\mathrm{Ag}(111)$ recorded at $V=1.2 \mathrm{~V}, I=20 \mathrm{pA}$. Inset: Line profile of a monolayer $\mathrm{MoS}_{2}$ island along the green line. b) Close-up view on the moiré structure. c) Atomically resolved terminating $S$ layer $(V=5 \mathrm{mV}, I=1 \mathrm{nA})$. d) Constant-height dl/dV spectra of $\mathrm{MoS}_{2} / \mathrm{Ag}(111)$ recorded on a top and on a hollow region of the moiré structure as shown on the inserted STM topography (feedback opened at $\left.V=2.5 \mathrm{~V}, I=0.5 \mathrm{nA}, V_{\text {mod }}=10 \mathrm{mV}\right)$. The inset shows the gap region of $\mathrm{MoS}_{2} / \mathrm{Ag}(111)$ on a logarithmic scale. We identify the valence band maximum (VBM) and the conduction band minimum (CBM) as the changes of the slope of the $\mathrm{d} / / \mathrm{d} V$ signal. Dashed lines indicate the CBM at approx. $0.05 \mathrm{~V}$ and the VBM at approx. $-1.55 \mathrm{~V}$. The strong features in the $\mathrm{d} / \mathrm{d} V$ spectra are associated to the onset of specific bands, which are labeled by $Q$, $\Gamma_{1}$ and $\Gamma_{2}$ according to their location in the Brillouin zone. The assignment follows that in [38]

pattern bears a topographic and an electronic modulation [38], we investigate the differential conductance $(\mathrm{d} I / \mathrm{d} V)$ spectra on different locations (Figure 1d). We first examine the spectrum on the top site of the moiré structure. We observe a gap in the density of states, which is flanked by an onset of conductance at approx. $-1.55 \mathrm{~V}$ and approx. $+0.05 \mathrm{~V}$ (marked by dashed lines labeled VBM or CBM, which have been determined from a logarithmically scaled plot). Additionally, there are pronounced peaks at approx. $0.77 \mathrm{~V}$ and approx. $1.28 \mathrm{~V}$. First, we note that the observed bandgap is significantly smaller than the $2.8 \mathrm{eV}$ bandgap of a single layer of free-standing $\mathrm{MoS}_{2}[30,31]$. This indicates a strong hybridization of the electronic states of the $\mathrm{MoS}_{2}$ layer and the Ag substrate. Second, we note that the spectral features are similar to those observed for single-layer $\mathrm{MoS}_{2}$ on $\mathrm{Au}(111)[29,35,38]$. For direct comparison, we plot the spec- tra on the top sites of the $\mathrm{MoS}_{2}$ moiré on $\mathrm{Au}(111)$ and $\mathrm{Ag}(111)$ in Figure 2a. At negative bias voltage, the onsets of conductance are essentially the same, while the features at positive bias voltage appear approx. $140 \mathrm{mV}$ closer to the Fermi level on $\mathrm{Ag}(111)$ than on $\mathrm{Au}(111)$.

Before discussing the differences between the layers on $\mathrm{Au}(111)$ and $\mathrm{Ag}(111)$, we investigate the effect of the different stacking at the interface on the electronic properties. The spectrum of a hollow site on $\operatorname{Ag}(111)$ shows a shift of the features at negative bias voltage by about approx. $130 \mathrm{mV}$ towards the Fermi level $\left(E_{\mathrm{F}}\right)$, whereas the peaks at positive bias undergo a much smaller shift (approx. $50 \mathrm{mV}$ ) away from $E_{\mathrm{F}}$ (Figure 1d). $\mathrm{On} \mathrm{Au}(111)$, there are also variations between hollow and top sites, with the strongest shift at negative bias voltage (Figure 2). a)

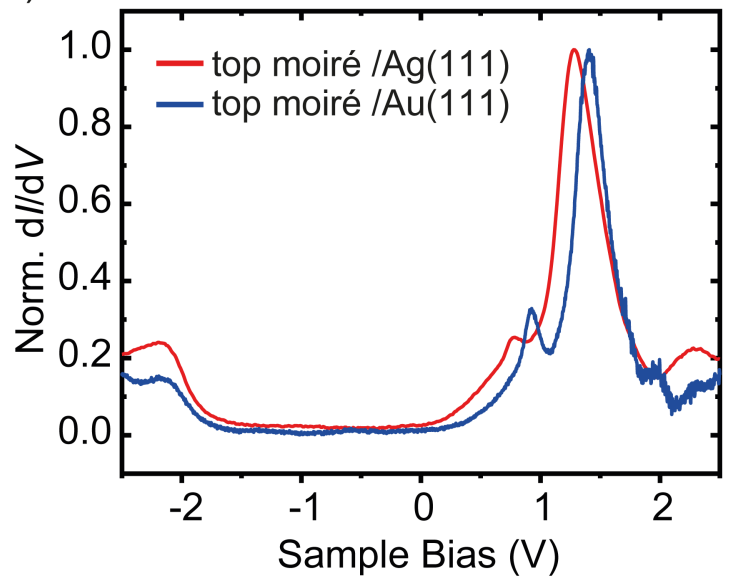

b)

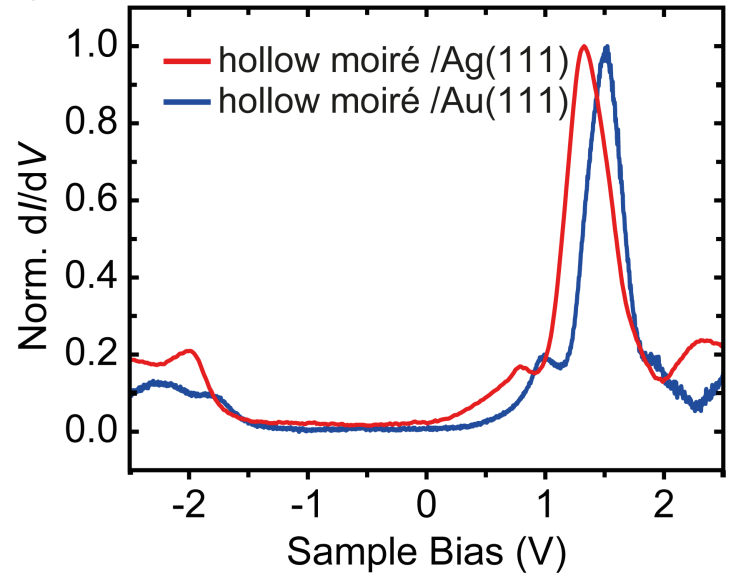

Figure 2: Constant-height $\mathrm{d} / \mathrm{d} V$ spectra recorded (a) on a top and (b) on a hollow site of the moiré structure of $\mathrm{MoS}_{2}$ on $\mathrm{Ag}(111)$ (red curves) and on $\mathrm{Au}(111)$ (blue curves). Feedback opened at $V=2.5 \mathrm{~V}, I=0.5 \mathrm{nA}, V_{\mathrm{mod}}=10 \mathrm{mV}$ (all spectra, except for hollow site on Au(111): $V_{\mathrm{mod}}=5 \mathrm{mV}$ ). 
To understand the differences between the substrate and the local sites, we first discuss the origin of the spectroscopic features. Based on the similarity of the spectral shapes on $\mathrm{Au}(111)$ and $\mathrm{Ag}(111)$, we tentatively assign the strong peaks at approx. $0.8 \mathrm{~V}$ (labeled as $\Gamma_{1}$ ) and approx. $1.3 \mathrm{~V}$ (labeled as $\Gamma_{2}$ ) (values averaged over the different moiré sites) to bands at the $\bar{\Gamma}$ point [38]. More precisely, the peak at $\Gamma_{2}$ has been assigned to bands at $\Gamma$, which are also present in free-standing $\mathrm{MoS}_{2}$, but are broadened due to hybridization with the substrate. The peak at $\Gamma_{1}$ has been observed in tunneling spectra of $\mathrm{MoS}_{2}$ on $\mathrm{Au}(111)$, but has not been found in calculations. It has been interpreted as a hybrid metal- $\mathrm{MoS}_{2}$ or an interface state [38]. The conduction band minimum, which is expected to lie at the $\bar{K}$ point for quasi free-standing as well as metal-supported single-layer $\mathrm{MoS}_{2}$ $[29,42-44]$, is hardly visible in the tunneling spectra due to the rapid decay of the tunneling constant with $k_{\|}[38,45]$. The same applies to the valence band maximum, such that the strongest feature in the tunneling spectra at $-2 \mathrm{~V}$ arises from bands close to the $\bar{Q}$ point [38].

Comparison of spectra on the moiré hollow sites suggest a rigid shift of the conduction bands between the $\mathrm{MoS}_{2}$ bands on $\mathrm{Ag}$ and $\mathrm{Au}$. In a very simple interpretation, this agrees with the lower work function of Ag than that of Au. A down-shift of the conduction band structure by approx. $280 \mathrm{meV}$ has been observed by photoemission of $\mathrm{WS}_{2}$ on $\mathrm{Au}(111)$ and $\mathrm{Ag}(111)$ [33]. Angle-resolved measurements further showed that the shift also included band distortions, such that bands at $Q$ were crossing $E_{\mathrm{F}}$ (instead of at $K$ ). The band distortion was explained by hybridization of the $\mathrm{WS}_{2}$ bands with the Ag substrate [33]. As our $\mathrm{d} I / \mathrm{d} V$ signal is not sensitive to $k \|$, we would not be able to detect band distortions in the $\mathrm{MoS}_{2}-\mathrm{Ag}$ system. However, the clear shift of the states at $\Gamma$ can be easily understood by hybridization of S-derived states of mainly out-of-plane character with Ag states in analogy to [29].

In the occupied states, the bands on the hollow site follow the same trend of a down-shift, suggesting that the states near $\bar{Q}$ are equally affected by hybridization with $\mathrm{Ag}$ states [33]. In contrast, the tunneling spectra on the top sites, seem to coincide for $\mathrm{Au}$ and $\mathrm{Ag}$ substrates. We also note that the tunneling conductance close to the $\bar{Q}$ point is the most sensitive to the precise location on the moiré pattern. Hence, we suggest that this site is most strongly affected by screening effects, which may vary on the different substrates [46] and partially compensate for hybridization effects.

\section{Electronic properties of TCNQ molecules on $\mathrm{MoS}_{2}$ on $\mathrm{Ag}(111)$}

Deposition of TCNQ molecules (structure shown in Figure 3a) on the sample held at $230 \mathrm{~K}$ leads to large densely packed mo- lecular islands on the $\mathrm{MoS}_{2}$ areas (Figure 3b). The large size and high degree of order of these islands reflects a low diffusion barrier on the $\mathrm{MoS}_{2}$ substrate. The moiré pattern of $\mathrm{MoS}_{2}$ remains intact and visible through the molecular monolayer. High-resolution STM images recorded at $0.8 \mathrm{~V}$ (Figure 3c) allow for the resolution of the individual molecules and their arrangement. Each TCNQ molecule appears with back-to-back double U-shapes separated by a nodal plane. As will be discussed later, and based on previous work on TCNQ [5,23], this appearance can be associated to the spatial distribution of the lowest unoccupied molecular orbital (LUMO). The molecular arrangement can be described by the lattice vectors $a_{1}=0.9 \pm 0.1 \mathrm{~nm}, a_{2}=1.0 \pm 0.1 \mathrm{~nm}$ and the angle $(96 \pm 2)^{\circ}$ (see model in Figure 3c). This structure is stabilized by dipole-dipole interactions between the cyano endgroups and the quinone center of neighboring molecules. This assembly is very similar to typical self-assembled TCNQ islands on weakly interacting substrates [5,23,47-49]. When measured at a lower bias voltage (e.g., at $V=0.2 \mathrm{~V}$ in Figure 4a), the molecules appear with featureless elliptical shape, reflecting only the topographic extent of the molecules.
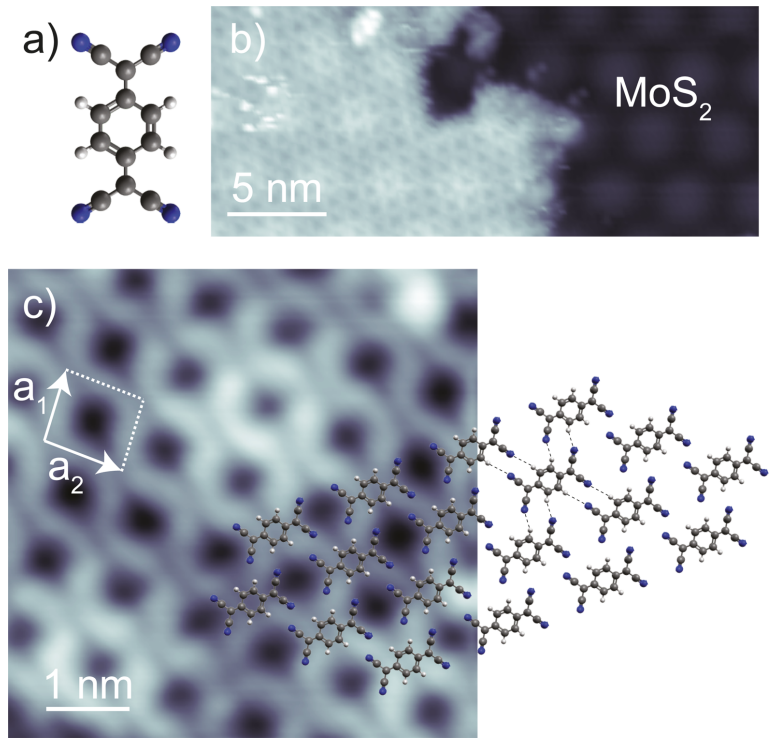

Figure 3: a) Stick-and-ball model of TCNQ. Gray, blue, and white spheres represent $\mathrm{C}, \mathrm{N}$, and $\mathrm{H}$ atoms, respectively. b) STM topography of a TCNQ molecular island on $\mathrm{MoS}_{2} / \mathrm{Ag}(111)$ recorded at $V=1 \mathrm{~V}$, $I=10 \mathrm{pA}$. c) STM topography of a TCNQ island on $\mathrm{MoS}_{2} / \mathrm{Ag}(111)$ recorded at $V=0.8 \mathrm{~V}, I=200 \mathrm{pA}$, with superimposed molecular models suggesting intermolecular dipole-dipole interactions (dashed lines). White arrows represent the unit cell of the self-organized TCNQ domain with lattice vectors $a_{1}=0.9 \pm 0.10 \mathrm{~nm}$ and $a_{2}=1.0 \pm 0.10 \mathrm{~nm}$ and the angle between them of $(96 \pm 2)^{\circ}$.

The strong bias-voltage dependence of the TCNQ molecules on the $\mathrm{MoS}_{2}$ layer promises energetically well separated molecular states. To investigate these properties in more detail, we re- 

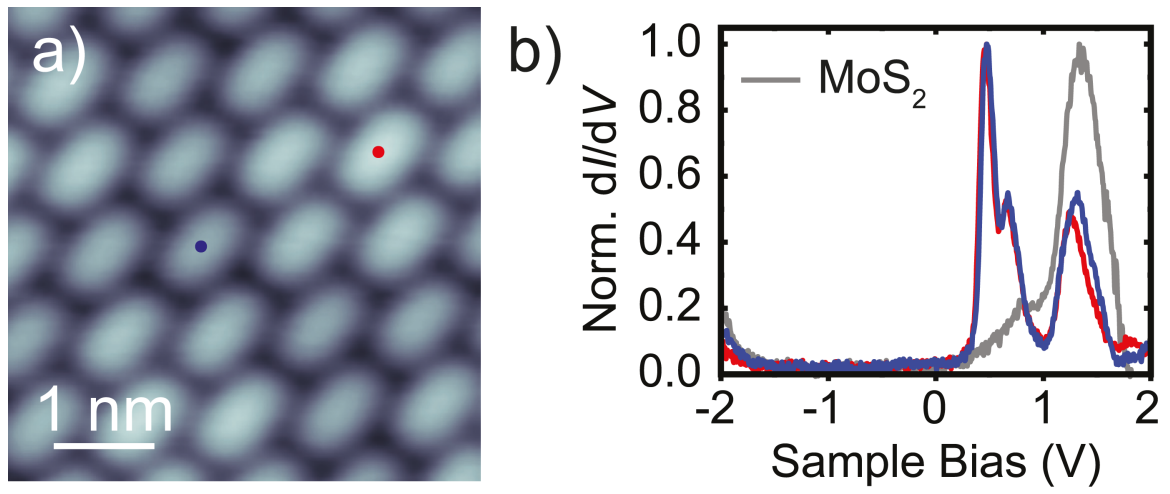

Figure 4: a) STM topography of a self-assembled TCNQ island on $\mathrm{MoS}_{2} / \mathrm{Ag}(111)$, recorded at $V=0.2 \mathrm{~V}, I=20 \mathrm{pA}$. b) dl/dV spectra acquired on $\mathrm{TCNQ}$ molecules within the island in panel a, with the precise location marked by colored dots. The gray spectrum was recorded on a bare MoS 2 layer for reference. Feedback opened at $V=2 \mathrm{~V}, I=100 \mathrm{pA}$, with $V_{\text {mod }}=20 \mathrm{mV}$.

corded $\mathrm{d} I / \mathrm{d} V$ spectra on top of the molecules (Figure $4 \mathrm{~b}$ ). These show two main resonances at approx. $0.47 \mathrm{~V}$ and approx. $0.64 \mathrm{~V}$. Another peak at approx. $1.3 \mathrm{~V}$ matches the $\Gamma$ resonance of the bare $\mathrm{MoS}_{2}$ layer. At negative bias voltage, we observe an onset of conductance at approx. $-1.8 \mathrm{~V}$. The $\mathrm{d} I / \mathrm{d} V$ spectra thus show that the STM image in Figure 4a was recorded within the energy gap of the molecule, which explains the featureless shape. In order to determine the origin of each of the resonances, we recorded constant-height $\mathrm{d} I / \mathrm{d} V$ maps at their corresponding energies (Figure 5).
For the first resonance at positive bias voltage $(470 \mathrm{mV}$, Figure 5a), we observe the same double U-shape, separated by a nodal plane, which we used in Figure 3 for the identification of the molecular arrangement. The $\mathrm{d} I / \mathrm{d} V$ map at $640 \mathrm{mV}$ exhibits the same shape, suggesting the same orbital as its origin. At $1.3 \mathrm{~V}$, the molecules do not show any characteristic feature (Figure 5c). Finally, Figure 5d presents a conductance map at $-2 \mathrm{~V}$ associated with the onset of conductance observed at negative bias voltage for spectra on the molecule. Here, the $\mathrm{d} I / \mathrm{d} V$ signal is rather blurred, but we remark that it is more
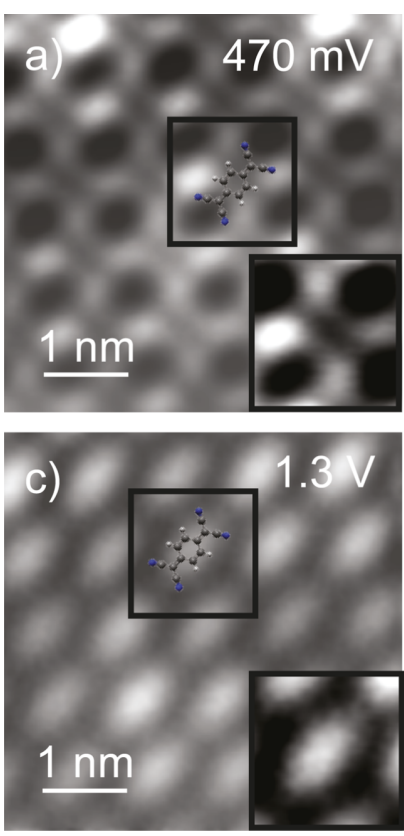
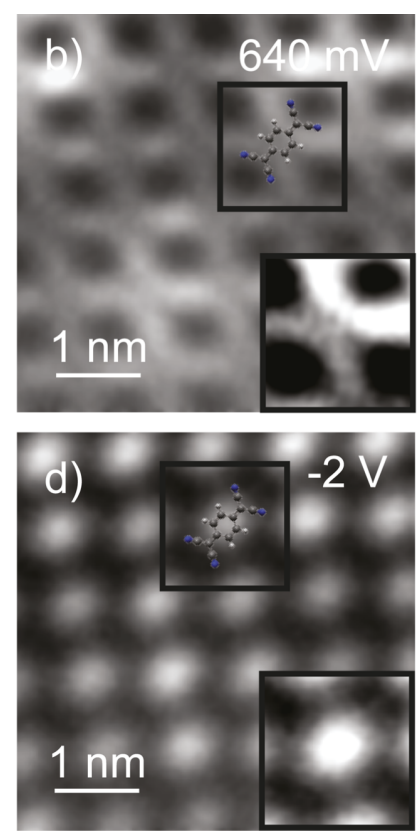

e)

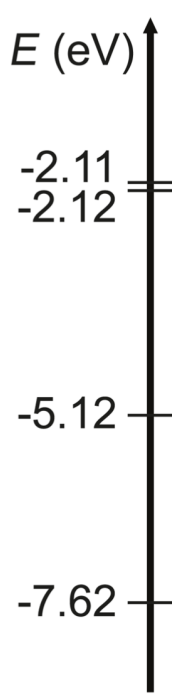

$\begin{array}{cc}\text { simulated } & \text { molecular } \\ \text { conductance } & \text { orbital }\end{array}$

map

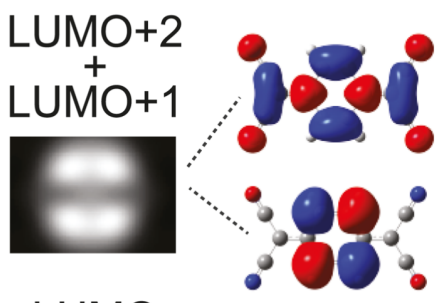

LUMO
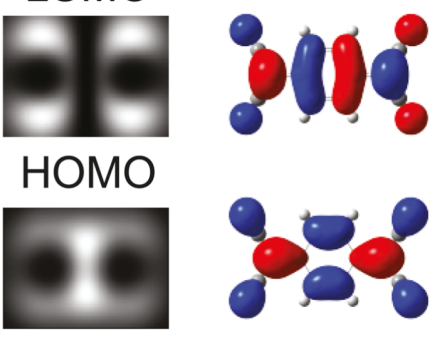

Figure 5: a-d) Constant-height $d / d V$ maps of a TCNQ island on $M_{2} S_{2}$ recorded at the resonance energies derived in Figure $4 \mathrm{~b}$. Feedback opened in panels (a-c) $V=2 \mathrm{~V}, I=100 \mathrm{pA}$ and (d) $V=-2 \mathrm{~V}, I=30 \mathrm{pA}$ on the center of the molecule with $V_{\mathrm{mod}}=20 \mathrm{mV}$. Close-up images with enhanced contrast on one molecule are shown as inset for each map. e) Energy-level diagram of TCNQ determined from gas-phase DFT calculations (left). The isosurfaces of the frontier molecular orbitals are shown on the right. These have been used to calculate the tunneling matrix element $M_{\mathrm{ts}}$ with an s-wave tip at a tip-molecule distance of $7.5 \AA$, work function of $5 \mathrm{eV}$. The map of the spatial distribution of $\left|M_{\mathrm{ts}}\right|^{2}$ is shown in the middle panel. 
localized in the center of the molecule as compared to the elliptical shape in Figure 5c.

For the identification of molecular orbitals, it is often sufficient to compare the $\mathrm{d} I / \mathrm{d} V$ maps with the shape of the gas-phase molecular orbitals. Using this method, the U-shaped features have previously been associated to the LUMO of TCNQ $[5,23,49]$. Here, we corroborate this assignment by simulating constantheight $\mathrm{d} I / \mathrm{d} V$ maps of a free, flat-lying molecule. We first calculated the gas-phase electronic structure using density functional theory (DFT) calculations with the B3PW91 functional and the 6-31 $\mathrm{g}(\mathrm{d}, \mathrm{p})$ basis set as implemented in the Gaussian 09 package [50]. The isodensity contour plots of the highest occupied molecular orbital (HOMO) and some of the lowest unoccupied orbitals are shown in Figure 5e, right panel. The HOMO/ LUMO can be unambiguously distinguished by the absence/ presence of a nodal plane at the center of the quinone backbone. For direct comparison with the $\mathrm{d} I / \mathrm{d} V$ maps, we calculate the tunneling matrix element between an s-wave tip and the spatially resolved molecular wave function across the molecule [51]. The maps of the square of the tunneling matrix element are depicted in Figure 5e next to the corresponding molecular orbitals. Because LUMO+1 and LUMO+2 are quasi-degenerate, we used the sum of their wave functions for the calculations of the tunneling matrix elements. As expected, the nodal planes of the molecular orbitals dominate the simulated $\mathrm{d} I / \mathrm{d} V$ maps and can be taken as a robust signature for molecular orbital identification. Additionally, the simulated maps reveal that the $\mathrm{d} I / \mathrm{d} V$ intensity is not directly proportional to the isosurface density. For instance, there is hardly any intensity within the $U$ shapes of the TCNQ LUMO, and the HOMO is mainly localized at the very center of the quinone moiety. We note that the simulated maps were obtained at a tip-molecule distance (center of the s-wave tip to center of the molecule) of $7.5 \AA$. This value was chosen because it represents reasonable tunneling conditions in experiments. However, variation of the tip height by $( \pm 2 \AA)$ does not have any influence on the observation of the main features within the map (i.e., nodal planes, or intensity maxima) [52].

Comparison with the experimental constant-height $\mathrm{d} I / \mathrm{d} V$ maps, now allows for an unambiguous identification of the origin of the molecular resonances. As suggested previously, the resonance at $0.47 \mathrm{~V}$ can be derived from the LUMO with the double U-shape being in very good agreement with the calculations of the tunneling matrix element. The very same signatures in the conductance map at $0.64 \mathrm{~V}$ suggest that this resonance stems from the LUMO as well. The DFT calculations show that the LUMO is non-degenerate. Hence, we can exclude a substrateinduced lifting of the degeneracy. The energy difference of only $170 \mathrm{meV}$ between the two resonances lies within the typical vibrational energies of organic molecules and may, thus, be indicative of a vibronic peak. We will elucidate this point further below.

The $\mathrm{d} I / \mathrm{d} V$ map at $1.3 \mathrm{~V}$ essentially shows the same elliptical shapes of the molecules as the STM image recorded in the electronic gap (Figure 4a). Our DFT calculations suggest that the next higher unoccupied orbitals lie $3 \mathrm{eV}$ above the LUMO and show a pattern of nodal planes that are absent in the experiment. Additionally, given the energy similar to that of the $\mathrm{MoS}_{2}$ bands, this resonance is probably not associated to the molecular layer, but to direct tunneling into the $\mathrm{MoS}_{2}$ states.

The assignment of the orbital origin at negative bias voltage bears some intricacies, because the experimental map lacks characteristic nodal planes. The reduced spatial resolution is most probably caused by the overlap with density of states of the substrate as we are approaching the onset of the valence band of $\mathrm{MoS}_{2}$. One may suggest that the stronger localization of $\mathrm{d} I / \mathrm{d} V$ intensity toward the quinone center is in agreement with the large tunneling matrix element of the HOMO at the center of the molecule. This assignment may be enforced by the coincidence of the observed molecular energy gap of TCNQ with the DFT-derived gap. However, DFT is known to underestimate HOMO-LUMO gaps. Although this effect may be compensated by the screening properties of the substrate, we refrain from a definite assignment. In any case, our data clearly shows that the HOMO is at or within the conduction band of $\mathrm{MoS}_{2}$.

By comparison with simulations, we thus arrive at a clear identification of the energy level alignment. Most notably, we find that the LUMO-derived resonance lies close to, but above, the Fermi level of the substrate, whereas the HOMO is far below. This leaves the molecule in a neutral state with a negligible amount of charge transfer, despite the electron accepting character of TCNQ. Nonetheless, its electron affinity of approx. $3.4 \mathrm{eV}[53,54]$ is consistent with the LUMO alignment just above $E_{\mathrm{F}}$ when considering the work function of $\mathrm{MoS}_{2} / \mathrm{Ag}(111)$ of $4.7 \mathrm{eV}$ [55]. We found small shifts of the LUMO onsets by at most $50 \mathrm{mV}$ between the spectra of TCNQ molecules lying at the top or hollow sites of the moiré structure of $\mathrm{MoS}_{2}$. These shifts correspond to the moiré-induced shifts in unoccupied states of the $\mathrm{MoS}_{2}$ layer and thus only reflect the different screening properties from the substrate. In turn, we do not observe any modification of the electronic structure of $\mathrm{MoS}_{2}$. This indicates weak interactions of the molecules all along the $\mathrm{MoS}_{2}$ layer.

Importantly, the resonance at $470 \mathrm{mV}$ has a rather narrow width of approx. $100 \mathrm{mV}$. This is significantly smaller than reso- 
nances typically observed on metal surfaces, where strong hybridization effects lead to widths of the order of approx. $500 \mathrm{meV}[5,48]$. The narrow width thus reflects that $\mathrm{MoS}_{2}$ acts as a decoupling layer from the metal substrate. However, this resonance width is broader than what has been observed for the HOMO resonance of other organic molecules on $\mathrm{MoS}_{2}$ on $\mathrm{Au}(111)[26,52,56]$. In contrast to those cases, where the HOMO lay well inside the electronic gap of $\mathrm{MoS}_{2}$, the LUMO of TCNQ is located right at the onset of the conduction band. This provides relaxation pathways for electrons tunneling into the LUMO, though still significantly less than on the bare metal.

\section{Vibronic excitations of TCNQ on $\mathrm{MoS}_{2}$ on $\mathrm{Ag}(111)$}

Having shown that the resonances at 470 and $640 \mathrm{mV}$ originate both from the LUMO of TCNQ, we now turn to a more detailed analysis. A close-up view of the spectral range with these peaks is shown in the bottom panel of Figure 6b with the LUMOderived peak at $470 \mathrm{mV}$ shifted to zero energy and its peak height being normalized. The satellite structure is reminiscent of vibronic sidebands, which occur due to the simultaneous excitation of a vibrational mode upon charging [22,25,57-61]. The sidepeaks should thus obey the same symmetry as the parent orbital state [62-64]. In the simplest case, these a)

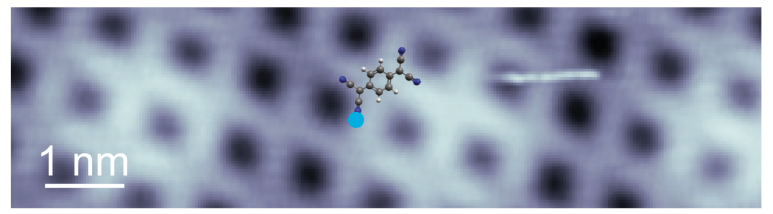

b)

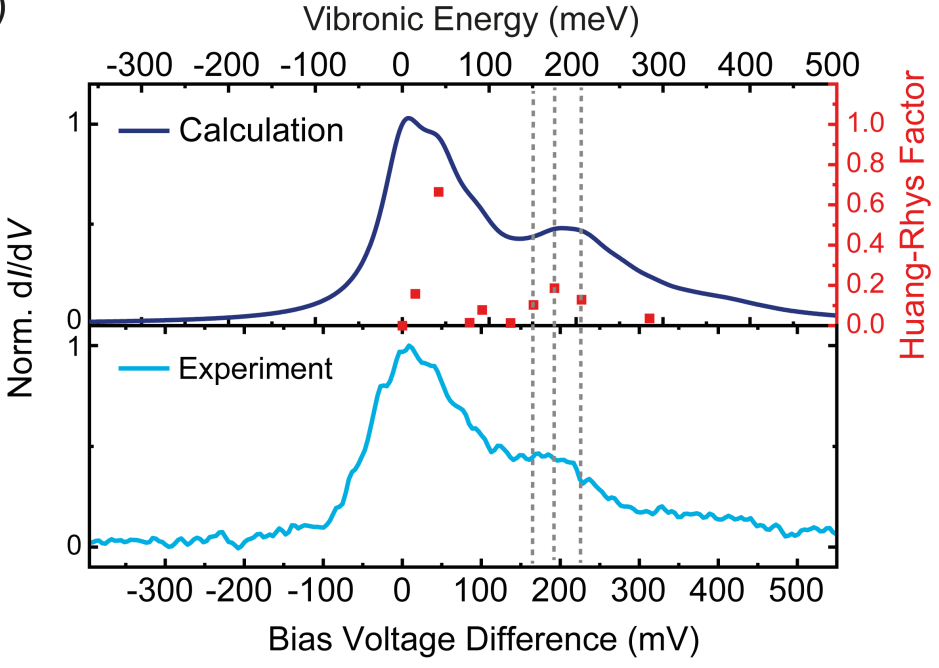

c)

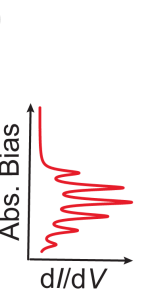

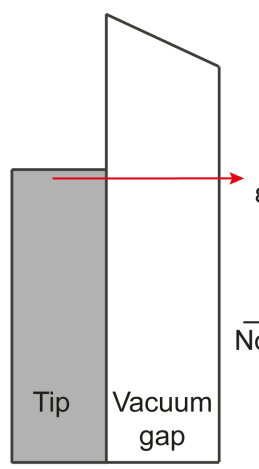

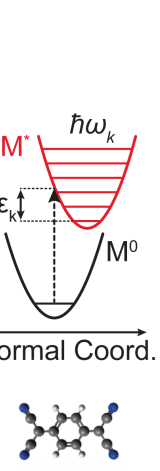

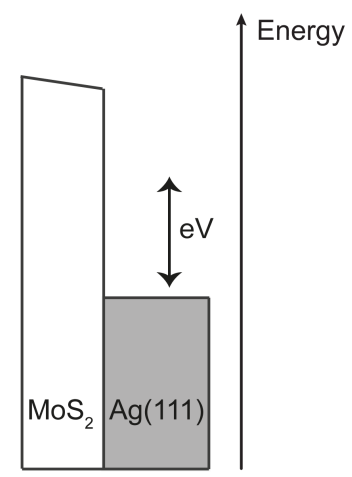

d)

Mode $150.9 \mathrm{meV}$

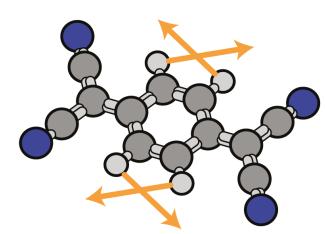

e)

Mode $175.0 \mathrm{meV}$

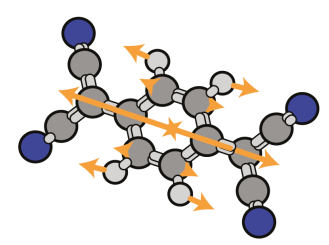

f)

Figure 6: a) STM topography image of a TCNQ island recorded at $V=1 \mathrm{~V}, I=10 \mathrm{pA}$. b) Simulated (top panel) and experimental (bottom panel) $\mathrm{d} / \mathrm{d} V$ spectra at the position indicated by the blue dot in panel (a) with feedback opened at $V=2 \mathrm{~V}, I=100 \mathrm{pA}$, with $V_{\text {mod }}=10 \mathrm{mV}$. The simulated spectrum is obtained from DFT calculations for all vibrational modes of the TCNQ- molecule with a Huang-Rhys factor higher than 0.01 (dots associated with the right axis). A Lorentzian peak of $60 \mathrm{meV}$ broadening is applied to all of these modes. c) Schematic representation of electron transport through a TCNQ molecule adsorbed on $\mathrm{MoS}_{2} / \mathrm{Ag}(111)$ : singly charged TCNQ- is formed upon injecting an electron into a vibronic state of an unoccupied molecular electronic level. $d-f$ ) Visualization of the vibrational modes contributing to the satellite peak. The orange arrows represent the displacement of the atoms involved in these vibrations. 
excitations can be described within the Franck-Condon model (see sketch in Figure 6c). When probing the LUMO in tunneling spectroscopy, the molecule is transiently negatively charged. Within the Born-Oppenheimer approximation, this process is described by a vertical transition in the energy level diagram from the ground state $M^{0}$ to the excited state $M^{*}$. Upon charging, the molecule undergoes a geometric distortion, captured by the shift of the potential energy curve of the excited state. Vertical transitions allow for probing many vibronic states, with the intensities given by a Poisson distribution,

$$
I_{k n}=e^{-S_{k}} \frac{S_{k}^{n}}{n !},
$$

with $S_{k}$ being the Huang-Rhys factor of the vibrational mode $k$ and $n$ its harmonics. The Huang-Rhys factor is determined by the relaxation energy $\varepsilon_{k}$ of a vibrational mode when charging the molecule as

$$
S_{k}=\frac{\varepsilon_{k}}{\hbar \omega_{k}} .
$$

From the DFT calculations of the TCNQ molecule, we determine all vibrational eigenmodes in the negatively charged state and also derive the Huang-Rhys factors $S_{k}$ [26]. The latter are plotted in the upper panel of Figure $6 \mathrm{~b}$ (dots, right axis). Applying to each of the vibronic states a Lorentzian peak with a full width at half maximum of $60 \mathrm{meV}$ and intensity proportional to the Poisson distribution, as described above, leads to the simulated Franck-Condon spectrum in the upper panel of Figure $6 \mathrm{~b}$. This spectrum closely resembles the experimental one and, therefore, nicely reflects the nature of the satellite structure. We note that the bias voltage axis (bottom panel) is scaled by $10 \%$ compared to the energy axis (top panel) to account for the voltage drop across the $\mathrm{MoS}_{2}$ layer [65]. We now realize that the peak at approx. $640 \mathrm{meV}$ consists of three vibrational modes (at 151, 175, and $206 \mathrm{meV}$ ) exhibiting a large Huang-Rhys factor. These modes correspond to in-plane breathing modes of TCNQ (see schemes in Figure 6d-f), which are particularly sensitive to charging. Additionally, a mode at $40 \mathrm{meV}$ has a large Huang-Rhys factor. The excitation of this mode is not energetically well separated from the elastic onset of the LUMO in experiment. However, this mode contributes to an asymmetric line shape, which can be realized by comparing the low-energy flank to the high-energy fall-off of the first resonance. The low-energy side can be fitted by a Voigt profile and suggests a lifetime broadening of $55 \pm 15 \mathrm{meV}$. This is, however, insufficient for a peak separation from the mode at $40 \mathrm{meV}$.
We further note that the experimental spectrum was taken on a cyano group, where no nodal planes exist in the LUMO, as their presence may lead to vibration-assisted tunneling in addition to the bare Franck-Condon excitation [52].

\section{Conclusion}

We have shown that a single layer of $\mathrm{MoS}_{2}$ may act as a decoupling layer for molecules from the underlying metal surface, if the molecular resonances lie within the semiconducting bandgap of $\mathrm{MoS}_{2}$. MoS 2 on $\mathrm{Au}(111)$ and $\mathrm{Ag}(111)$ exhibit very similar gap structures, but are shifted in energy according to the different work functions of the metal. Though this is not the only reason for the band modifications [33], we suggest that such considerations may help when searching for appropriate decoupling layers for specific molecules. We have challenged the decoupling properties of $\mathrm{MoS}_{2} / \mathrm{Ag}(111)$ for TCNQ molecules. These exhibit their LUMO resonance just at the conduction band onset of $\mathrm{MoS}_{2}$, whereas the HOMO lies within the valence band. Hence, the HOMO is not decoupled from the substrate, and also the LUMO suffers considerable lifetime broadening as compared to resonances, which would be well separated from the onsets of the $\mathrm{MoS}_{2}$ bands. The lifetime broadening of $55 \pm 15 \mathrm{meV}$ can be translated into a lifetime of approx. $6 \mathrm{fs}$ of the excited state. This is almost one order of magnitude longer than on the bare metal surface, where the hot electron vanishes into the bulk on ultrafast timescales, but an order of magnitude shorter than for molecular resonances well separated from the band onsets $[26,52,56]$. Yet, the increase in the lifetime of the excited state allowed us to resolve vibronic states of the transiently negatively charged TCNQ molecule albeit only up to approx. $200 \mathrm{meV}$ above the LUMO resonance, where contributions of $\mathrm{MoS}_{2}$ bands at $\Gamma$ become strong. Our simulations reproduce the experimental satellite structure of the LUMO very well, although the experimental width prevented us from resolving the individual modes.

\section{Acknowledgements}

We acknowledge discussions with S. Trishin and J. R. Simon.

\section{Funding}

A. Yousofnejad acknowledges a scholarship from the Claussen-Simon Stiftung. This work was supported by the Deutsche Forschungsgemeinschaft (DFG) - project number 182087777 - SFB 951 (A14), and project number 328545488 TRR 227 (B05).

\section{ORCID ${ }^{\circledR}$ iDs}

Asieh Yousofnejad - https://orcid.org/0000-0002-7275-0228

Gaël Reecht - https://orcid.org/0000-0001-5036-7271

Christian Lotze - https://orcid.org/0000-0001-6847-5676

Katharina J. Franke - https://orcid.org/0000-0001-9416-023X 


\section{Preprint}

A non-peer-reviewed version of this article has been previously published as a preprint https://arxiv.org/abs/2006.12154

\section{References}

1. Lu, X.; Grobis, M.; Khoo, K. H.; Louie, S. G.; Crommie, M. F. Phys. Rev. B 2004, 70, 115418. doi:10.1103/physrevb.70.115418

2. Thygesen, K. S.; Rubio, A. Phys. Rev. Lett. 2009, 102, 046802. doi:10.1103/physrevlett.102.046802

3. Braun, S.; Salaneck, W. R.; Fahlman, M. Adv. Mater. (Weinheim, Ger.) 2009, 21, 1450-1472. doi:10.1002/adma.200802893

4. Tautz, F. S. Prog. Surf. Sci. 2007, 82, 479-520. doi:10.1016/j.progsurf.2007.09.001

5. Torrente, I. F.; Franke, K. J.; Pascual, J. I. Int. J. Mass Spectrom. 2008, 277, 269-273. doi:10.1016/j.ijms.2008.07.013

6. Tegeder, P. J. Phys.: Condens. Matter 2012, 24, 394001. doi:10.1088/0953-8984/24/39/394001

7. Reed, M. A.; Zhou, C.; Muller, C. J.; Burgin, T. P.; Tour, J. M. Science 1997, 278, 252-254. doi:10.1126/science.278.5336.252

8. Reichert, J.; Ochs, R.; Beckmann, D.; Weber, H. B.; Mayor, M.; v. Löhneysen, H. Phys. Rev. Lett. 2002, 88, 176804. doi:10.1103/physrevlett.88.176804

9. Venkataraman, L.; Klare, J. E.; Tam, I. W.; Nuckolls, C.; Hybertsen, M. S.; Steigerwald, M. L. Nano Lett. 2006, 6, 458-462. doi:10.1021/nl052373+

10. Lafferentz, L.; Ample, F.; Yu, H.; Hecht, S.; Joachim, C.; Grill, L. Science 2009, 323, 1193-1197. doi:10.1126/science.1168255

11. Nitzan, A.; Ratner, M. A. Science 2003, 300, 1384-1389. doi:10.1126/science. 1081572

12. Tao, N. J. Nat. Nanotechnol. 2006, 1, 173-181. doi:10.1038/nnano.2006.130

13. Aradhya, S. V.; Venkataraman, L. Nat. Nanotechnol. 2013, 8, 399-410. doi:10.1038/nnano.2013.91

14. Reecht, G.; Lotze, C.; Sysoiev, D.; Huhn, T.; Franke, K. J. ACS Nano 2016, 10, 10555-10562. doi:10.1021/acsnano.6b06559

15. Repp, J.; Meyer, G.; Stojković, S. M.; Gourdon, A.; Joachim, C. Phys. Rev. Lett. 2005, 94, 026803. doi:10.1103/physrevlett.94.026803

16. Liljeroth, P.; Repp, J.; Meyer, G. Science 2007, 317, 1203-1206. doi:10.1126/science.1144366

17. Qiu, X. H.; Nazin, G. V.; Ho, W. Science 2003, 299, 542-546. doi:10.1126/science.1078675

18. Heinrich, A. J.; Gupta, J. A.; Lutz, C. P.; Eigler, D. M. Science 2004, 306, 466-469. doi:10.1126/science.1101077

19. Rau, I. G.; Baumann, S.; Rusponi, S.; Donati, F.; Stepanow, S.; Gragnaniello, L.; Dreiser, J.; Piamonteze, C.; Nolting, F.; Gangopadhyay, S.; Albertini, O. R.; Macfarlane, R. M.; Lutz, C. P.; Jones, B. A.; Gambardella, P.; Heinrich, A. J.; Brune, H. Science 2014, 344, 988-992. doi:10.1126/science.1252841

20. Hirjibehedin, C. F.; Lutz, C. P.; Heinrich, A. J. Science 2006, 312, 1021-1024. doi:10.1126/science.1125398

21. Franke, K. J.; Schulze, G.; Henningsen, N.; Fernández-Torrente, I.; Pascual, J. I.; Zarwell, S.; Rück-Braun, K.; Cobian, M.; Lorente, N. Phys. Rev. Lett. 2008, 100, 036807. doi:10.1103/physrevlett.100.036807

22. Matino, F.; Schull, G.; Köhler, F.; Gabutti, S.; Mayor, M.; Berndt, R. Proc. Natl. Acad. Sci. U. S. A. 2011, 108, 961-964. doi:10.1073/pnas.1006661107
23. Garnica, M.; Stradi, D.; Barja, S.; Calleja, F.; Díaz, C.; Alcamí, M.; Martín, N.; Vázquez de Parga, A. L.; Martín, F.; Miranda, R. Nat. Phys. 2013, 9, 368-374. doi:10.1038/nphys2610

24. Riss, A.; Wickenburg, S.; Tan, L. Z.; Tsai, H.-Z.; Kim, Y.; Lu, J.; Bradley, A. J.; Ugeda, M. M.; Meaker, K. L.; Watanabe, K.; Taniguchi, T.; Zettl, A.; Fischer, F. R.; Louie, S. G.; Crommie, M. F. ACS Nano 2014, 8, 5395-5401. doi:10.1021/nn501459v

25. Schulz, F.; Drost, R.; Hämäläinen, S. K.; Liljeroth, P. ACS Nano 2013, 7, 11121-11128. doi:10.1021/nn404840h

26. Krane, N.; Lotze, C.; Reecht, G.; Zhang, L.; Briseno, A. L.; Franke, K. J. ACS Nano 2018, 12, 11698-11703. doi:10.1021/acsnano.8b07414

27. Repp, J.; Meyer, G.; Paavilainen, S.; Olsson, F. E.; Persson, M. Phys. Rev. Lett. 2005, 95, 225503. doi:10.1103/physrevlett.95.225503

28. Fatayer, S.; Schuler, B.; Steurer, W.; Scivetti, I.; Repp, J.; Gross, L.; Persson, M.; Meyer, G. Nat. Nanotechnol. 2018, 13, 376-380. doi:10.1038/s41565-018-0087-1

29. Bruix, A.; Miwa, J. A.; Hauptmann, N.; Wegner, D.; Ulstrup, S.; Grønborg, S. S.; Sanders, C. E.; Dendzik, M.; Grubišić Čabo, A.; Bianchi, M.; Lauritsen, J. V.; Khajetoorians, A. A.; Hammer, B.; Hofmann, P. Phys. Rev. B 2016, 93, 165422. doi:10.1103/physrevb.93.165422

30. Cheiwchanchamnangij, T.; Lambrecht, W. R. L. Phys. Rev. B 2012, 85, 205302. doi:10.1103/physrevb.85.205302

31. Qiu, D. Y.; da Jornada, F. H.; Louie, S. G. Phys. Rev. Lett. 2013, 111, 216805. doi:10.1103/physrevlett.111.216805

32. Bana, H.; Travaglia, E.; Bignardi, L.; Lacovig, P.; Sanders, C. E.; Dendzik, M.; Michiardi, M.; Bianchi, M.; Lizzit, D.; Presel, F.; De Angelis, D.; Apostol, N.; Kumar Das, P.; Fujii, J.; Vobornik, I.; Larciprete, R.; Baraldi, A.; Hofmann, P.; Lizzit, S. $2 D$ Mater. 2018, 5 , 035012. doi:10.1088/2053-1583/aabb74

33. Dendzik, M.; Bruix, A.; Michiardi, M.; Ngankeu, A. S.; Bianchi, M.; Miwa, J. A.; Hammer, B.; Hofmann, P.; Sanders, C. E. Phys. Rev. B 2017, 96, 235440. doi:10.1103/physrevb.96.235440

34. Grønborg, S. S.; Ulstrup, S.; Bianchi, M.; Dendzik, M.; Sanders, C. E.; Lauritsen, J. V.; Hofmann, P.; Miwa, J. A. Langmuir 2015, 31, 9700-9706. doi:10.1021/acs.langmuir.5b02533

35. Krane, N.; Lotze, C.; Läger, J. M.; Reecht, G.; Franke, K. J. Nano Lett. 2016, 16, 5163-5168. doi:10.1021/acs.nanolett.6b02101

36. Wakabayashi, N.; Smith, H. G.; Nicklow, R. M. Phys. Rev. B 1975, 12, 659-663. doi:10.1103/physrevb.12.659

37. Sørensen, S. G.; Füchtbauer, H. G.; Tuxen, A. K.; Walton, A. S.; Lauritsen, J. V. ACS Nano 2014, 8, 6788-6796. doi:10.1021/nn502812n

38. Krane, N.; Lotze, C.; Franke, K. J. Surf. Sci. 2018, 678, 136-142. doi:10.1016/j.susc.2018.03.015

39. Bronsema, K. D.; De Boer, J. L.; Jellinek, F. Z. Anorg. Allg. Chem. 1986, 540, 15-17. doi:10.1002/zaac.19865400904

40. Schumacher, A.; Scandella, L.; Kruse, N.; Prins, R. Surf. Sci. 1993, 289, L595-L598. doi:10.1016/0039-6028(93)90875-k

41. Helveg, S.; Lauritsen, J. V.; Lægsgaard, E.; Stensgaard, I.; Nørskov, J. K.; Clausen, B. S.; Topsøe, H.; Besenbacher, F. Phys. Rev. Lett. 2000, 84, 951-954. doi:10.1103/physrevlett.84.951

42. Mak, K. F.; Lee, C.; Hone, J.; Shan, J.; Heinz, T. F. Phys. Rev. Lett. 2010, 105, 136805. doi:10.1103/physrevlett.105.136805

43. Splendiani, A.; Sun, L.; Zhang, Y.; Li, T.; Kim, J.; Chim, C.-Y.; Galli, G.; Wang, F. Nano Lett. 2010, 10, 1271-1275. doi:10.1021/nl903868w

44. Miwa, J. A.; Ulstrup, S.; Sørensen, S. G.; Dendzik, M.; Čabo, A. G.; Bianchi, M.; Lauritsen, J. V.; Hofmann, P. Phys. Rev. Lett. 2015, 114, 046802. doi:10.1103/physrevlett.114.046802 
45. Zhang, C.; Chen, Y.; Johnson, A.; Li, M.-Y.; Li, L.-J.; Mende, P. C.; Feenstra, R. M.; Shih, C.-K. Nano Lett. 2015, 15, 6494-6500. doi:10.1021/acs.nanolett.5b01968

46. Rösner, M.; Steinke, C.; Lorke, M.; Gies, C.; Jahnke, F.; Wehling, T. O. Nano Lett. 2016, 16, 2322-2327. doi:10.1021/acs.nanolett.5b05009

47. Barja, S.; Garnica, M.; Hinarejos, J. J.; Vázquez de Parga, A. L.; Martín, N.; Miranda, R. Chem. Commun. 2010, 46, 8198-8200. doi:10.1039/c0cc02675a

48. Park, C.; Rojas, G. A.; Jeon, S.; Kelly, S. J.; Smith, S. C.; Sumpter, B. G.; Yoon, M.; Maksymovych, P. Phys. Rev. B 2014, 90 , 125432. doi:10.1103/physrevb.90.125432

49. Pham, V. D.; Ghosh, S.; Joucken, F.; Pelaez-Fernandez, M.; Repain, V.; Chacon, C.; Bellec, A.; Girard, Y.; Sporken, R.; Rousset, S.; Dappe, Y. J.; Narasimhan, S.; Lagoute, J. npj 2D Mater. Appl. 2019, 3, 5. doi:10.1038/s41699-019-0087-5

50. Gaussian 09, Revision D.01; Gaussian, Inc.: Wallingford, CT, USA, 2009.

51. Bardeen, J. Phys. Rev. Lett. 1961, 6, 57-59. doi:10.1103/physrevlett.6.57

52. Reecht, G.; Krane, N.; Lotze, C.; Zhang, L.; Briseno, A. L.; Franke, K. J. Phys. Rev. Lett. 2020, 124, 116804. doi:10.1103/physrevlett.124.116804

53. Milián, B.; Pou-Amérigo, R.; Viruela, R.; Ortı, E. Chem. Phys. Lett. 2004, 391, 148-151. doi:10.1016/j.cplett.2004.04.102

54. Zhu, G.-Z.; Wang, L.-S. J. Chem. Phys. 2015, 143, 221102. doi:10.1063/1.4937761

55. Zhong, H.; Quhe, R.; Wang, Y.; Ni, Z.; Ye, M.; Song, Z.; Pan, Y.; Yang, J.; Yang, L.; Lei, M.; Shi, J.; Lu, J. Sci. Rep. 2016, 6, 21786. doi:10.1038/srep21786

56. Reecht, G.; Krane, N.; Lotze, C.; Franke, K. J. ACS Nano 2019, 13, 7031-7035. doi:10.1021/acsnano.9b02117

57. Qiu, X. H.; Nazin, G. V.; Ho, W. Phys. Rev. Lett. 2004, 92, 206102. doi:10.1103/physrevlett.92.206102

58. Pradhan, N. A.; Liu, N.; Ho, W. J. Phys. Chem. B 2005, 109, 8513-8518. doi:10.1021/jp045289b

59. Nazin, G. V.; Wu, S. W.; Ho, W. Proc. Natl. Acad. Sci. U. S. A. 2005, 102, 8832-8837. doi:10.1073/pnas.0501171102

60. Frederiksen, T.; Franke, K. J.; Arnau, A.; Schulze, G.; Pascual, J. I.; Lorente, N. Phys. Rev. B 2008, 78, 233401. doi:10.1103/physrevb.78.233401

61. Wickenburg, S.; Lu, J.; Lischner, J.; Tsai, H.-Z.; Omrani, A. A.; Riss, A.; Karrasch, C.; Bradley, A.; Jung, H. S.; Khajeh, R.; Wong, D.; Watanabe, K.; Taniguchi, T.; Zettl, A.; Neto, A. H. C.; Louie, S. G.; Crommie, M. F. Nat. Commun. 2016, 7, 13553. doi:10.1038/ncomms13553

62. Huan, Q.; Jiang, Y.; Zhang, Y. Y.; Ham, U.; Ho, W. J. Chem. Phys. 2011, 135, 014705. doi:10.1063/1.3598958

63. Schwarz, F.; Wang, Y. F.; Hofer, W. A.; Berndt, R.; Runge, E.; Kröger, J. J. Phys. Chem. C 2015, 119, 15716-15722. doi:10.1021/acs.jpcc.5b03392

64. Mehler, A.; Néel, N.; Bocquet, M.-L.; Kröger, J. J. Phys.: Condens. Matter 2019, 31, 065001. doi:10.1088/1361-648x/aaf54c

65. Krane, N.; Lotze, C.; Bogdanoff, N.; Reecht, G.; Zhang, L.; Briseno, A. L.; Franke, K. J. Phys. Rev. B 2019, 100, 035410. doi:10.1103/physrevb.100.035410

\section{License and Terms}

This is an Open Access article under the terms of the Creative Commons Attribution License (http://creativecommons.org/licenses/by/4.0). Please note that the reuse, redistribution and reproduction in particular requires that the authors and source are credited.

The license is subject to the Beilstein Journal of Nanotechnology terms and conditions: (https://www.beilstein-journals.org/bjnano)

The definitive version of this article is the electronic one which can be found at:

doi:10.3762/bjnano.11.91 